\title{
Ultrasonography versus computed tomography for initial investigation of suspected nephrolithiasis
}

\author{
Reviewed by: Katie Lin, MD*; Shawn Dowling, MD* ${ }^{{ }^{\dagger \ddagger}}$
}

Clinical question

Is ultrasonography a safe and practical alternative to computed tomography for the initial investigation of suspected nephrolithiasis?

Article chosen

Smith-Bindman R, Aubin C, Bailitz J, et al. Ultrasonography versus computed tomography for suspected nephrolithiasis. N Engl J Med 2014;371(12):1100-10.

Objective

To assess the safety and efficacy of ultrasonography as a first-line imaging investigation for patients with suspected nephrolithiasis.

Keywords: computed tomography, diagnostic imaging, kidney calculi, nephrolithiasis, ultrasonography, ureteric calculi

\section{BACKGROUND}

In 2014, Smith-Bindman et al. published a paper in the New England fournal of Medicine titled: "Ultrasonography versus computed tomography for suspected

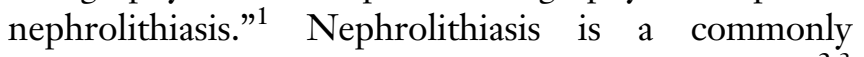
encountered diagnosis in the emergency department. ${ }^{2,3}$ It is associated with significant patient morbidity and health care cost. ${ }^{1}$ Abdominal computed tomography (CT) has become the most common first-line imaging modality for investigating nephrolithiasis. ${ }^{4,5}$

CT is attractive due to its high sensitivity for stone detection, ability to assess stone size, ${ }^{6,7}$ and utility in making alternate diagnoses. The main limitations of CT include exposure to ionizing radiation, a high rate of incidental findings, increased cost per investigation, and its lack of demonstrated correlation with improved patient outcomes. ${ }^{8-11}$

Ultrasonography, which is readily available in most emergency departments, ${ }^{12}$ presents a radiation-sparing alternative for the investigation of suspected nephrolithiasis and, in addition, has point-of-care applications. However, image quality is user-dependent and can be limited by body habitus. The purpose of the reviewed study is to explore the utility of ultrasonography as an alternative to CT for initial assessment of suspected nephrolithiasis.

\section{POPULATION STUDIED}

Smith-Bindman et al. ${ }^{1}$ enrolled adult patients (18-76 years old) with flank or abdominal pain who were imaged for suspected nephrolithiasis. Exclusion criteria were pregnancy, obesity (defined in men as $>129 \mathrm{~kg}$, women $>113 \mathrm{~kg}$ ), single kidney, prior renal transplant, dialysis, and high risk for serious alternative diagnoses (e.g., acute cholecystitis, appendicitis, aortic aneurysm, bowel disorders), as determined by the treating physician.

\section{STUDY DESIGN}

This was a prospective, randomized, multicentre, unblinded, comparative effectiveness trial involving 15 academic emergency departments across the United States.

Enrolled patients were assigned randomly to one of three imaging groups in 1:1:1 fashion: 1) point-of-care ultrasonography (POCUS) performed at the bedside by

From the *Department of Emergency Medicine, University of Calgary, Calgary, AB; †Foothills Medical Centre, Calgary, AB; and $¥ A$ lberta Children’s Hospital, Calgary, AB

Correspondence to: Dr. Katie Lin, Foothills Medical Centre, Department of Emergency Medicine, Room C231, 1403-29 St. NW, Calgary, AB, T2N 2T9; Email: kt.yuwei.lin@gmail.com 
an emergency physician with training, as recommended by the American College of Emergency Physicians (ACEP); 2) formal (radiology) ultrasonography, performed by ultrasound technicians and/or radiologists in the radiology department; or 3) abdominal CT. Subsequent follow-up imaging was obtained as necessary based on the judgment of the treating physician.

Diagnostic accuracy was determined by the comparison of discharge diagnosis with later confirmation of nephrolithiasis by either patient reported observation of stone passage or surgical stone removal or lithotripsy. The primary analysis was by intentionto-treat; however, sensitivity and specificity were calculated and reported by both intention-to-treat principles and according to the first imaging test chosen and actually obtained.

\section{OUTCOMES MEASURED}

The primary outcomes included 30-day incidence of high-risk diagnoses with complications related to missed or delayed diagnosis (predefined by conditions such as ruptured abdominal aortic aneurysm), 6-month cumulative radiation exposure, and cost.

Secondary outcomes included serious adverse events (predefined as events that resulted in death or threat of death, admission, significant disability, or requirement for intervention), pain score, rates of return emergency department visits, admission rates, and diagnostic accuracy.

\section{STUDY RESULTS}

A total of 3638 patients were screened, and 2759 were randomized and included in the intention-to-treat population. There were 908 patients in the POCUS group, 893 in the radiology ultrasonography group, and 958 in the CT group. Baseline characteristics were similar between the groups. There was an overall loss to follow-up of 113 patients (4.1\%), with similar rates between groups. Follow-up occurred at 3, 7, 30, 90, and 180 days after randomization through telephone and structured interviews.

With respect to the study's primary objectives, no statistically significant difference between groups was found for 30-day high-risk diagnoses with complications $(0.7 \%$ in the POCUS group, $0.3 \%$ in the radiology ultrasonography group, and $0.2 \%$ in the CT group, $p=0.30)$. Cumulative radiation exposure over
6 months was significantly lower in the POCUS and radiology ultrasonography groups as compared to the CT group (10.1 mSv, $9.3 \mathrm{mSv}$, and $17.2 \mathrm{mSv}$, respectively, $p<0.001)$. Cost outcomes were incompletely reported and not fully analysed.

No statistically significant differences were found between study groups for any of the secondary outcomes, with the exception of length of stay in the emergency department. Median length of stay was longer in the radiology ultrasonography group than either the POCUS group or the CT group ( 7 hours, 6.3 hours, and 6.4 hours, respectively, $p<0.001$ ).

The intention-to-treat analysis yielded no significant differences between the three groups for diagnostic accuracy; however, calculations based only on the first imaging investigation chosen (rather than intentionto-treat analysis) showed that both POCUS and radiology ultrasonography had lower sensitivities and higher specificities as compared to CT as first-line imaging modalities $(54 \%, 57 \%$, and $88 \%$ for sensitivity, respectively; $71 \%, 73 \%$, and $58 \%$ for specificity, respectively).

\section{COMMENTARY}

Current guidelines are sparse regarding initial imaging of suspected nephrolithiasis, and no published recommendations exist from either the American Urological Association or the Canadian Urological Association. The results of this study suggest that ultrasonography may be a safe and appropriate first-line imaging alternative to the more commonly used CT.

This was the first large scale, randomized, comparative effectiveness study powered to evaluate safety outcomes in the context of ultrasonography versus CT for suspected nephrolithiasis. The sample population was recruited from 15 American hospitals and was reflective of Canadian adult populations in the emergency department, with the noted exception of slight differences in ethnic distributions. ${ }^{13}$ An unblinded model was employed for feasibility reasons, as well as to permit additional imaging at the discretion of the treating physician according to standard practice. Statistical analysis followed intention-to-treat principles with additional calculations by the authors to assess diagnostic accuracy based only on the first imaging test chosen.

This study supports the safety of ultrasonography as a first-line imaging test in suspected nephrolithiasis. The majority of patients $(59.3 \%$ of POCUS patients 
and $73 \%$ of radiology ultrasound patients) who received ultrasonography as first-line imaging for suspected nephrolithiasis were successfully and safely spared from radiation exposure. A total of $40.7 \%$ of patients in the POCUS group and $27 \%$ of patients in the radiology ultrasound group had subsequent CT studies. There were no statistically significant differences found in 30-day high-risk complication rates, serious adverse events, subjective pain scores, or rates of return visits to the emergency department. Providing that physicians pursued follow-up imaging as required, there appeared to be no significant difference in adverse outcomes.

One limitation of the study was its lack of POCUS exam standardization. The POCUS exam was stated to follow ACEP ultrasound guidelines ${ }^{14}$; however, there was no outlined mechanism to ensure regulation of technique or quality control.

The low overall event rate (11 cases) for the primary outcome of 30-day high-risk diagnoses with complications merits mention. Although it is possible that this reflects a true low adverse event rate for diagnostic workup of suspected nephrolithiasis, it does raise potential concerns for insufficient capture of important negative outcomes and thus a resulting loss of power, either due to the small sample size, deaths in the loss to follow-up group, or insufficient outcome follow-up period. Missed high-risk diagnoses with complications are reported in Table S2 of the supplementary appendix. ${ }^{1}$ The POCUS group had the highest event rate with three cases of pyelonephritis with urosepsis, one case of urosepsis alone, one case of diverticulitis with abscess, and one case of small bowel obstruction with ischemia requiring resection. The radiology ultrasound group had one case of pyelonephritis, one case of renal abscess, and one case of ovarian torsion. The CT group had one case of pyelonephritis with urosepsis and one case of urosepsis alone. Diagnostic delay for missed diagnoses ranged from 2-30 days. There were five reported deaths within the study; all occurred $>30$ days after randomization and were not deemed to be related to study participation.

Although this study provides useful data on the comparative accuracy of ultrasonography versus CT, it was not specifically designed with appropriate power for sensitivity and specificity calculations. The intention-totreat analysis falsely elevates accuracy calculations for ultrasonography due to the significant proportion of patients who received dual-imaging and therefore had positive diagnoses based on the subsequent CT scan results. Accuracy evaluation based only on first imaging is thus arguably more clinically relevant, and showed ultrasound to have a significantly lower sensitivity but higher specificity for nephrolithiasis, as compared to CT.

\section{CONCLUSION}

This was a large-scale investigation with important implications for Canadian emergency departments. The study's results provide reassurance regarding the safety of ultrasound as an alternative to CT in first-line imaging for suspected nephrolithiasis. Even with subsequent imaging as required, more than half of patients who underwent initial ultrasonography in this study required no follow-up CT scans and were not found to experience any statistically significant increased risk of complications related to missed or delayed diagnoses.

This study was not designed or powered to provide definitive conclusions about sensitivity or specificity of ultrasonography versus CT. Ultrasound is a notably operator-dependent tool, and further studies would be necessary to validate the findings in this area. However, the reported safety results for first-line ultrasonography are encouraging, especially for physicians seeking to minimize radiation exposure in younger patients or who practice in centres with limited CT access.

Acknowledgements: We extend thanks to Tiffany Junghans and Dr. Eddy Lang for providing editorial guidance.

Competing interests: None declared.

\section{REFERENCES}

1. Smith-Bindman R, Aubin C, Bailitz J, et al. Ultrasonography versus computed tomography for suspected nephrolithiasis. N Engl 7 Med 2014;371(12):1100-10.

2. Ghani KR, Roghmann F, Sammon JD, et al. Emergency department visits in the United States for upper urinary tract stones: trends in hospitalization and charges. $f$ Urol 2014;191(1):90-6.

3. Scales CD Jr, Smith AC, Hanley JM, et al. Prevalence of kidney stones in the United States. Eur Urol 2012;62(1):160-5.

4. Coursey CA, Casalino DD, Reimer EM, et al. ACR Appropriateness Criteria ${ }^{\circledR}$ acute onset flank painsuspicion of stone disease. Ultrasound Q 2012;28(3):227-33.

5. Tasian GE, Pulido JE, Keren R, et al. Use of and regional variation in initial CT imaging for kidney stones. Pediatrics 2014;134(5):909-15.

6. Heneghan JP, McGuire KA, Leder RA, et al. Helical CT for nephrolithiasis and ureterolithiasis: comparison of conventional and reduced radiation-dose techniques. Radiology 2003;229(2):575-80. 
7. Demehri S, Kalra MK, Rybicki FJ, et al. Quantification of urinary stone volume: attenuation threshold-based CT method-a technical note. Radiology 2011;258(3):915-22.

8. Fahmy NM, Elkoushy MA, Andonian S. Effective radiation exposure in evaluation and follow-up of patients with urolithiasis. Urology 2012;79(1):43-7.

9. Thompson RJ, Wojcik SM, Grand WD, et al. Incidental findings on CT scans in the emergency department. Emerg Med Int 2011;2011:624847.

10. Westphalen AC, Hsia RY, Maselli JH, et al. Radiological imaging of patients with suspected urinary tract stones: national trends, diagnoses, and predictors. Acad Emerg Med 2011;18(7):699-707.

11. Rai BP, Ali A, Raslan M, et al. Fate of indeterminate lesions detected on noncontrast computed tomography scan for suspected urolithiasis: a retrospective cohort study with a minimum follow-up of 15 months. Urology 2014;84(6): 1272-4.

12. Torreggiani WC, Nicolaou S, Lyburn ID, et al. Emergency radiology in Canada: a national survey. Can Assoc Radiol 7 2002;53(3):160-7.

13. Statistics Canada. National Household Survey, 2011: Immigration and ethnocultural diversity in Canada; 2011. Available at: http://www12.statcan.gc.ca/nhs-enm/2011/as-sa/ 99-010-x/99-010-x2011001-eng.pdf (accessed 4 January 2015).

14. American College of Emergency Physicians. Policy statement: emergency ultrasound imaging criteria compendium; 2014. Available at: http://www.acep.org/workarea/ downloadasset.aspx?id=32886 (accessed 17 March 2015). 